\title{
Convergence of mean curvature flows with surgery
}

\author{
Joseph Lauer
}

\begin{abstract}
Huisken and Sinestrari [9] have recently defined a surgery process for mean curvature flow when the initial data are a two-convex hypersurface in $\mathbb{R}^{n+1}(n \geq 3)$. The process depends on a parameter $H$. Its role is to initiate a surgery when the maximum of the mean curvature of the evolving hypersurface becomes $H$, and to control the scale at which each surgery is performed. We prove that as $H \rightarrow \infty$ the surgery process converges to level set flow $[1,2]$.
\end{abstract}

\section{Introduction}

Huisken and Sinestrari [9] have recently defined a mean curvature flow with surgery when the initial data are a two-convex hypersurface in $\mathbb{R}^{n+1}$ when $n \geq 3$. The process depends on a parameter $H$ ( $H_{3}$ in the notation of [9]), which controls both the maximal mean curvature and the scale at which each surgery is performed. In this note we investigate to what extent the process depends on this parameter.

Recall that a smooth one-parameter family of hypersurface immersions $F_{t}: M \rightarrow \mathbb{R}^{n+1}$ is a solution to mean curvature flow if

$$
\frac{\partial F}{\partial t}(x, t)=\vec{H}(F(x, t)),
$$

where $\vec{H}$ is the mean curvature vector. The first results were obtained by Huisken [8] who proved that if the initial data are convex and $n \geq 2$, then the mean curvature flow shrinks the hypersurface to a round point. The analogous result for curves in the plane $(n=1)$ was proved by Gage and Hamilton [3], and shortly after Grayson [4] showed that any embedded curve in the plane evolves to become convex. This means that the classification of singularities is particularly simple for embedded plane curves. However, when $n>1$ Grayson's Theorem no longer holds and singularities other than round points may occur. The existence of such a singularity was first proved rigourously by Grayson [5], who gave the example of a barbell-like surface which develops a neck-pinch. 
As an evolving hypersurface becomes singular the maximum of the mean curvature is unbounded, and hence constructing a surgery procedure requires detailed information about the geometry of the hypersurface in regions of high curvature. In the two-convex case Huisken and Sinestrari prove that such regions are diffeomorphic to $S^{n}$ or $S^{n-1} \times S^{1}$, and are discarded during surgery, or are neck-like regions in which the surgery replaces a topological cylinder by a pair of convex disks. As the parameter $H$ increases the surgeries are performed closer to the singular time and on quantitatively thinner necks. The detailed estimates in [9] controlling the length and width of the necks allow us to prove:

Theorem 1.1. Huisken-Sinestrari surgery converges to the level set flow as $H \rightarrow \infty$.

Since the limit is unique this result can be interpreted as a stability theorem for level set flow. Our approach is to use a barrier argument: we prove that for any $\epsilon>0$ there exists $H>0$, so that the mean curvature flow with surgery performed with parameter $H$ is disjoint (in space-time) from the level set flow of the initial hypersurface shifted backwards in time by $\epsilon$.

This result has also been obtained independently by J. Head [7] using new integral estimates for mean curvature and a careful consideration of the number of surgery times. While the proof here is a quicker route to Theorem 1.1, the methods of [7] provide bounds for the rate of convergence.

Since the Ricci flow with surgery constructed for 3-manifolds (see [11, 12]) also depends on a parameter, it is possible to consider the same question there. One obstacle in this direction is that there is not such a natural candidate for the limiting object.

\section{Weak notions of mean curvature flow}

In this section we recall (see $[9,10,13])$ two ways in which the evolution of a smooth hypersurface can be extended beyond a singularity: Level set flow and mean curvature flow with surgery.

Definition 2.1 Weak Set Flow. Let $K \subset \mathbb{R}^{n+1}$ be closed, and let $\left\{K_{t}\right\}_{t \geq 0}$ be a one-parameter family of closed sets with initial condition $K_{0}=K$ such that the space-time track $\cup\left(K_{t} \times\{t\}\right) \subset \mathbb{R}^{n+2}$ is closed. Then $\left\{K_{t}\right\}_{t \geq 0}$ is weak set flow for $K$ if for every smooth mean curvature flow $\Sigma_{t}$ defined on $[a, b]$ we have

$$
K_{a} \cap \Sigma_{a}=\emptyset \Longrightarrow K_{t} \cap \Sigma_{t}=\emptyset
$$

for each $t \in[a, b]$. 
It is essentially the definition that weak set flows avoid smooth mean curvature flows when the initial conditions are disjoint but a stronger statement is true: The distance between a weak set flow and a smooth mean curvature flow is nondecreasing in $t$. Otherwise, one could translate the initial data in space and obtain a contradiction to the definition of a weak set flow.

Definition 2.2 level set flow. The level set flow of a compact set $K \subset$ $\mathbb{R}^{n+1}$ is the maximal weak set flow. That is, a one-parameter family of closed sets $K_{t}$ with $K_{0}=K$ such that if $\widetilde{K}_{t}$ is any weak set flow with $\widetilde{K}_{0}=K$ then $\widetilde{K}_{t} \subset K_{t}$ for each $t \geq 0$.

The existence of a maximal weak set flow is verified by taking the closure of the union of all weak set flows with a given initial data. If $K_{t}$ is the level set flow of $K$, we denote by $\widehat{K}$ by the space-time track swept out by $K_{t}$. That is,

$$
\widehat{K}=\bigcup_{t \geq 0} K_{t} \times\{t\} \subset \mathbb{R}^{n+2} .
$$

The level set flow was introduced independently by Evans and Spruck [2] and Chen et al. [1]. It was first formulated in terms of viscosity solutions of partial differential equations whereas the geometric definition above was first used by Ilmanen [10].

Another approach to constructing weak solutions to geometric evolution equations has been to use a surgery procedure. This idea was first used by Hamilton [6] to avoid the development of singularities in Ricci flow.

Definition 2.3 surgery [9]. A mean curvature flow with surgery consists of the following data:

(1) An initial smooth hypersurface $\Sigma \subset \mathbb{R}^{n+1}$.

(2) Constants $\omega_{1}<\omega_{2}<1$ and $H>0$.

(3) A finite collection of times $0<t_{1}<t_{2} \ldots<t_{m}$ called surgery times (let $\left.t_{0}=0\right)$.

(4) A collection of mean curvature flows $\Sigma_{t}^{i}$ on $\left[t_{i}, t_{i+1}\right]$, with $\Sigma_{0}^{0}=\Sigma$, such that for each $i$ the maximum mean curvature on $\Sigma_{t}^{i}$ is $H$ and is achieved only when $t=t_{i+1}$.

(5) A surgery algorithm that consists of two steps:

(i) At each surgery time a finite number of necks with mean curvature greater than $\omega_{1} H$ are removed from $\Sigma_{t_{i+1}}^{i}$ and replaced with convex caps with mean curvature bounded by $\omega_{2} H$. The operation of 
replacing a single neck with two convex caps is called a standard surgery.

(ii) Finitely many components of the hypersurface constructed in (i) are removed. These components are recognized as being diffeomorphic to either $S^{n-1} \times S^{1}$ or $S^{n}$.

The result of the surgery algorithm is a smooth hypersurface $\Sigma_{t_{i+1}}^{i+1}$ with mean curvature bounded by $\omega_{2} H$.

We denote by $\Sigma_{H} \subset \mathbb{R}^{n+2}$ the space-time track swept-out by the hypersurfaces, and say that $\Sigma_{H}$ is a mean curvature flow with surgery performed with parameter $H$.

The main result of [9] is that a mean curvature flow with surgery can be constructed when the initial data are a closed immersed two-convex hypersurface of dimension at least three. Recall that a hypersurface $\Sigma \subset \mathbb{R}^{n+1}$ is two-convex if the sum of the two smallest principal curvatures is everywhere non-negative. It is proved that for any such initial data there exist $\omega_{1}$, $\omega_{2}$ and $H_{0}>0$, so that the surgery may be performed with any parameter $H \geq H_{0}$. In particular, $\omega_{1}$ and $\omega_{2}$ can be fixed independently of $H$. It is also shown that if the initial data are embedded then the hypersurface remains embedded even after a surgery time.

In this paper, we consider only embedded initial data since otherwise the level set flow will not coincide with the evolving hypersurface for small $t>0$.

It will be convenient to work with the regions bounded by the evolving hypersurface. Let $K \subset \mathbb{R}^{n+1}$ be a compact domain such that $\partial K$ is a smooth two-convex hypersurface. Then if $\partial K_{H}$ is a mean curvature flow with surgery we define $K_{H} \subset \mathbb{R}^{n+2}$ to be the region of space-time such that the $t=T$ time-slice of $K_{H}$ is the compact domain bounded by $\left(\partial K_{H}\right)_{T}$. The hypersurface $\left(\partial K_{H}\right)_{t}$ may not be connected after the first surgery time. However, the domains bounded by the connected components of $\left(\partial K_{H}\right)_{t}$ will be disjoint so that $\left(K_{H}\right)_{t}$ is well defined. Thus $K_{H}$ is an evolution of a union of domains whose boundary is a mean curvature flow with surgery performed with parameter $H$ in the sense defined above. We will also refer to $K_{H}$ as a mean curvature flow with surgery.

Notation 2.4. If $K_{H}$ is a mean curvature flow with surgery, and $T$ is a surgery time, then we use $\left(\partial K_{H}\right)_{T}^{-}$and $\left(\partial K_{H}\right)_{T}^{+}$to refer to the pre- and post-surgery hypersurfaces at time $T$, and $\left(K_{H}\right)_{T}^{-}$and $\left(K_{H}\right)_{T}^{+}$to refer to the regions they bound. 
If $K$ is a compact domain and $K_{H}$ is a mean curvature flow with surgery constructed in [9], then the cutting portion of the standard surgery algorithm only removes points in $\left(K_{H}\right)_{T}^{-}$. That is, the solid necks along which a standard surgery is performed are contained in the evolving domain. It follows that $K_{H}$ is a weak set flow for $K$. Note that this is not true if we consider only the evolving hypersurfaces, i.e., $\partial K_{H}$ is not a weak set flow of $\partial K$.

\section{Convergence}

In this section, we prove the convergence to level set flow. Theorem 3.1 is a more precise statement of Theorem 1.1 from the Introduction. Recall that $\widehat{K}$ denotes the space-time track of the level set flow of $K$.

Theorem 3.1. Let $K \subset \mathbb{R}^{n+1}, n \geq 3$, be a compact domain with $\partial K a$ smooth embedded two-convex hypersurface. For $H$ sufficiently large let $K_{H} \subset$ $\mathbb{R}^{n+2}$ be the result of the Huisken-Sinestrari surgery performed with parameter $H$, and initial condition $\left(K_{H}\right)_{0}=K$. Then

$$
\lim _{H \rightarrow \infty} K_{H}=\widehat{K}
$$

Remark 3.2. Convergence is with respect to the Hausdorff topology on closed sets in $\mathbb{R}^{n+2}$.

Theorem 3.1 follows from the following lemma regarding the surgery procedure, and a barrier argument. As usual, $B_{\epsilon}(x) \subset \mathbb{R}^{n+1}$ represents the ball of radius $\epsilon$ centered at $x$.

Lemma 3.3. Given $\epsilon>0$ there exists $H_{0}>0$, such that if $H \geq H_{0}, T$ is a surgery time, and $x \in \mathbb{R}^{n+1}$, then

$$
B_{\epsilon}(x) \subset\left(K_{H}\right)_{T}^{-} \Longrightarrow B_{\epsilon}(x) \subset\left(K_{H}\right)_{T}^{+} .
$$

The proof of Lemma 3.3 requires geometric information regarding the necks along which a surgery is performed. The parameter $H$ here corresponds to $H_{3}$ in [9], and $\omega_{1}, \omega_{2}$ are the constants appearing in Definition 2.3. Define $H_{1}=\omega_{1} H$ and $H_{2}=\omega_{2} H$. Furthermore, $\epsilon_{0}, k, \Lambda$ are defined in [9] and depend only on the initial hypersurface.

Proof of Lemma 3.3. Consider $K_{H}$ for $H \geq H_{0}$ where $H_{0}$ is a constant to be defined below. 
Since $T$ is a surgery time the algorithm defined in [9] identifies a finite collection of subsets, $\left\{A_{i}\right\}_{i=1}^{m}$, which cover the regions of $\left(\partial K_{H}\right)_{T}^{-}$with mean curvature greater than $\mathrm{H}_{2}$. There are three possibilities for the structure of the $A_{i}$ 's depending on whether each has 0,1 or 2 boundary components.

If $\partial A_{i} \neq \emptyset$ then for each component of $\partial A_{i}$ a standard surgery is performed. According to [9] there exists an embedding $N: S^{n-1} \times[a, b] \rightarrow A_{i}$ with strong geometric properties. In particular, $\Sigma_{z}=N\left(S^{n-1} \times z\right)$ has constant mean curvature in the image of $N$. We note that if $\partial A_{i}$ consists of two connected components then the map $N$ is a diffeomorphism. In general, $\partial A_{i}$ contains at least one of $\Sigma_{a}$ or $\Sigma_{b}$ and the mean curvature on $\partial A_{i}$ is $\frac{H_{1}}{2}$.

Suppose $\Sigma_{a} \subset \partial A_{i}$. We consider the standard surgery corresponding to $\Sigma_{a}$. Let $z_{0} \in[a, b]$ be the point closest to $a$ such that the mean curvature on $\Sigma_{z_{0}}$ is $H_{1}$. The slice $\Sigma_{z_{0}}$ is sufficiently far from $\partial A_{i}$ in the sense that $a<z_{0}-$ $4 \Lambda<z_{0}+4 \Lambda<b$, where $\Lambda \geq 10$. For simplicity, we will assume that $z_{0}=0$. The map $N$ can be extended (after first restricting it to $S^{n-1} \times[-4 \Lambda, 4 \Lambda]$ ) to a local diffeomorphism

$$
G: B_{1}^{n} \times[-4 \Lambda, 4 \Lambda] \rightarrow \mathbb{R}^{n+1},
$$

which is $\epsilon_{0}$-close in the $C^{k+1}$-norm to the standard isometric embedding of some tube $B^{n} \times[-4 \Lambda, 4 \Lambda]$ in $\mathbb{R}^{n+1}$ [[9], Prop. 3.25]. The standard surgery removes $N\left(S^{n-1} \times[-3 \Lambda, 3 \Lambda]\right)$ and replaces it by two convex caps contained in $G\left(B_{1}^{n} \times[-3 \Lambda, 3 \Lambda]\right)$, and the result is again a smooth embedded hypersurface [[9], Thm. 3.26]. By the Jordan-Brouwer Separation Theorem for hypersurfaces it follows that if $x \in\left(K_{H}\right)_{T}^{-} \backslash G\left(B_{1}^{n} \times[-3 \Lambda, 3 \Lambda]\right)$ then $x$ will remain in the interior of the hypersurface after the standard surgery.

Since $G$ is $\epsilon_{0}$-close to a standard tube and $\Lambda \geq 10$ is sufficiently large compared to $\epsilon_{0}$ we can choose $H_{0}$ large enough (and hence the radius of the tube small enough), so that

$$
B_{\epsilon}(x) \subset\left(K_{H}\right)_{T}^{-} \Longrightarrow B_{\epsilon}(x) \cap G\left(B_{1}^{n} \times[-3 \Lambda, 3 \Lambda]\right)=\emptyset .
$$

With this choice of $H_{0}$, we have that any $B_{\epsilon}(x) \subset\left(K_{H}\right)_{T}^{-}$remains in the interior of the hypersurface after a standard surgery. Note that at each surgery time a finite number of standard surgeries may be performed. However, the solid tubes associated to the surgeries are disjoint and so the surgeries do not interact.

It remains to verify that components discarded by 5)ii) of Definition 2.3 do not bound a ball of radius $\epsilon$. There are three ways in which such a component can arise:

(1) If $\partial A_{i}=\emptyset$ then $A_{i}$ is diffeomorphic to $S^{n}$ or $S^{n-1} \times S^{1}$ and is discarded. 
(2) If $\partial A_{i}$ consists of a single component then $A_{i}$ is homeomorphic to a ball. This corresponds to the case where the curvature does not decrease significantly in one direction along the neck. In this case only one standard surgery is performed. After the standard surgery, the end of the cylinder with high curvature will have become diffeomorphic to $S^{n}$ and will be discarded.

(3) If $\partial A_{i}$ consists of two components then a standard surgery is performed for each boundary component and the result is two capped cylinders and a component diffeomorphic to $S^{2}$. The $S^{2}$ component is discarded.

In each case the construction in [9] guarantees that the mean curvature of the component being removed is bounded from below by $\frac{H_{1}}{2}$. Suppose $\Sigma$ is such a hypersurface, that $x$ lies in the region bounded by $\Sigma$ and that $d=d(x, \Sigma) \geq \epsilon$. If $y \in \Sigma$ realizes $d(x, \Sigma)$ then the mean curvature at $y$ is not more than $\frac{n}{d} \leq \frac{n}{\epsilon}$ since $\Sigma \cap \operatorname{int}\left(B_{d}(x)\right)=\emptyset$. This is a contradiction as long as $H_{0} \geq \frac{2 n}{\epsilon \omega_{1}}$.

Proof of Theorem 3.1. Given $\epsilon>0$ sufficiently small let $t_{\epsilon}>0$ be the time such that

$$
d\left(\partial K, \partial K_{t_{\epsilon}}\right)=\epsilon
$$

Such a time exists because $\partial K$ is two-convex. Let $\Omega_{\epsilon} \subset \mathbb{R}^{n+2}$ be the level set flow of $K_{t_{\epsilon}}$. Equivalently, $\Omega_{\epsilon}$ is the level set flow of $K$ shifted backwards in time by $t_{\epsilon}$ (ignoring $t<0$ ).

Let $H_{0}=H_{0}(\epsilon)$ be chosen as in Lemma 3.3.

Claim: $\Omega_{\epsilon} \subset K_{H}$ for all $H \geq H_{0}$.

Let $T$ be the first surgery time of $K_{H}$. Since $\partial K_{H}$ is a smooth mean curvature flow on $[0, T)$ and $\Omega_{\epsilon}$ is weak set flow the distance between the two is nondecreasing on that interval, which implies

$$
d\left(\left(\Omega_{\epsilon}\right)_{T},\left(\partial K_{H}\right)_{T}^{-}\right) \geq \epsilon .
$$

Applying Lemma 3.3 we conclude that

$$
d\left(\left(\Omega_{\epsilon}\right)_{T},\left(\partial K_{H}\right)_{T}^{+}\right) \geq \epsilon .
$$

Since $\left(\partial K_{H}\right)_{T}^{+}$is a smooth hypersurface the argument can be repeated for each of the subsequent surgery times. This proves the claim.

Since $\lim _{\epsilon \rightarrow 0} \Omega_{\epsilon}=\widehat{K}$, the claim implies that $\widehat{K} \subset \lim _{H \rightarrow \infty} K_{H}$ as the limit of closed sets is closed. Note that the limit on the right-hand side exists since $H_{1}<H_{2}$ implies $K_{H_{1}} \subset K_{H_{2}}$. Finally, since $K_{H}$ is a weak set flow for $K$ the limit is also and thus $\lim _{H \rightarrow \infty} K_{H} \subset \widehat{K}$. 


\section{Acknowledgements}

The author wishes to thank Bruce Kleiner for suggesting the problem, and for his help and guidance during work on this project.

\section{References}

[1] Y.G. Chen, Y. Giga and S. Goto, Uniqueness and existence of viscosity solutions of generalized mean curvature flow equations, J. Differential Geom. 33(3) (1991), 749-786.

[2] L.C. Evans and J. Spruck, Motion of level sets by mean curvature. I, J. Differential Geom. 33(3) (1991), 635-681.

[3] M. Gage and R. Hamilton, The heat equation shrinking convex plane curves, J. Differential Geom. 23 (1986), 69-96.

[4] M. Grayson, The heat equation shrinks embedded plane curves to round points, J. Differential Geom. 26 (1987), 285-314.

[5] M. Grayson, A short note on the evolution of a surface by its mean curvature, Duke Math. J. 58(3) (1989), 555-558.

[6] R. Hamilton, Four-manifolds with positive isotropic curvature, Comm. Anal. Geom. 5 (1997), 1-92.

[7] J. Head, The surgery and level-set approaches to mean curvature flow, PhD Thesis, (2011), http://www.diss.fu-berlin.de/diss/receive/ FUDISS_thesis_000000024660.

[8] G. Huisken, Shrinking convex spheres by their mean curvature, J. Differential Geom. 20 (1984), 237-266.

[9] G. Huisken and C. Sinestrari, Mean curvature with surgery for twoconvex hypersurfaces, Invent. Math. 175 (2009), 137-221.

[10] T. Ilmanen, Elliptic regularization and partial regularity for motion by mean curvature, Mem. Amer. Math. Soc. 108(520) (1994), x+90.

[11] B. Kleiner and J. Lott, Notes on Perelman's papers, Geom. Topol. 12(5) (2008), 2587-2855.

[12] G. Perelman, Ricci flow with surgery on three-manifolds, arxiv: math.DG/0303109. 
[13] B. White, The size of the singular set in mean curvature flow of meanconvex sets, J. Amer. Math. Soc. 13(3) (2000), 665-695.

Department of Mathematics

YALE UNIVERSITY

NEW HAVEN

CT 06510

E-mail address: lauer@math.mit.edu

Received March 30, 2011 
